\title{
35
}

\section{Round the clock work: the technology issues}

\author{
Doug Vogel, University of Arizona, Tucson, Arizona USA, Chair
}

\section{PANEL FOCUS}

The issues to be explored in this panel represent the technological challenges associated with effective and efficiently supporting the international office of the future distributed in time and space. The sampling presented is indicative of this broad spectrum. Key issues are grouped as audio/video, computer hardware, connectivity/data-telecommunications, control software, and application software. More emerged through discussion.

\section{A SAMPLING OF ISSUES}

\subsection{Audio/Video}

Audio and video technologies vary significantly in characteristics and impact on group dynamics. The options and choices are many, including: document cameras for transparencies and opaque materials; video tape recorders; audio cassettes and compact discs; videodisc; $35 \mathrm{~mm}$ slides; television, satellite, or cable tuners; microphone pickup of all participant contributions for reinforcement at the same site and transmission to other sites; a stereo audio playback system for audio and video sources; large screen display of video and computer sources; scan conversion of computer text/graphics to video for recording; capture of video images for use by application software; life-size display of fixed cameras from other sites to give a "reach out and touch someone" effect. Which of these technologies and/or in what combination(s) provide the best return on investment given group and task characteristics is a perennial question.

\subsection{Computer hardware}

The hardware needs to effectively support the broader aspects of the IOF can become extensive. Multiple live video window capabilities integrated with the windowing of computer text/graphics may be needed to enhance users' interface with the system. Capture of textual documents through optical character recognition (OCR) is highly desirable, as is capture of graphic documents in a high resolution format. FAX transfer between sites or to other locations would aid in the incorporation of existing documents into some processes especially when other hardware is absent. When possible, the information FAXed should be in the form of a file rather than a physical document for ease of incorporation and highest resolution transfer. The capability to do "on-screen" annotation over computer graphics, text, or video facilitates the discussion of important issues. 


\subsection{Connectivity/Data-telecommunications}

To robustly interconnect sites, a number of communications channels may be required. The types of information to be transmitted over the different channels include combinations of audio, video, and graphics in electronic meeting system (EMS) environments. For example, a configuration may include: a fixed wide-angle camera shot of all participants, a mix of all participants' audio contributions, participant close-up shots and accompanying audio, a high resolution graphics image, carry network data transmissions, and equipment control data transmissions. Easy access to external resources e.g., the World Wide Web, is imperative. Issues quickly extend beyond technological aspects to encompass coordination between multiple telecommunications formats and protocols that span international boundaries.

\subsection{Control software}

To realize a truly unified environment, tight integration of graphics and presentation software with computer and traditional video, audio, and other technologies is essential. What is needed is a consistent interface between integrated information technology software and the wide range of information and presentation technologies from a variety of vendors that potentially could be incorporated. From the user's perspective, examples of the types of interfaces that need to be considered are: keyboards, mice, touch screens, light pens, graphics tablets, and voice recognition. The type of interface used will vary with each individual's preferences. Executives, educators and other professionals, the primary users of these facilities, tend to prefer "low touch" and "no touch" interfaces. Providing these types of interfaces may be essential to productivity and wide-based multi-cultural acceptance.

\subsection{Application software}

Requirements for effective distributed support software in the IOF includes aspects of individual, group, project, and organizational support all built upon a communications infrastructure.

Individual support needs include ready access to personal data sources and a degree of independence appropriate for personal exploration of topic areas. Group support includes the combination of session structure and process support seen in some groupware products. Project support includes presentation of the status and effective integration of information across sessions and between groups, thereby providing anelement of synergism and value added for a group engaged in a series of sessions. Organizational support includes access to internal organizational information as well as external information more commonly associated with executive support systems. This helps provide a level of information support that extends across projects.

\section{PANEL AIM}

The aim of the panel was to discuss these technological challenges associated with effectively and efficiently supporting the international office of the future and, in the process, identify others. With the set of technological challenges identified and understood, the panel discussion turned to strategies for dealing with them in different circumstances. The panel resulted in some early views as to appropriate technological infrastructures to support future work. 


\section{POSTSCRIPT - THE PANEL OUTCOMES}

The audience involvement using GroupSystems and subsequent discussion developed focus on three primary areas of technological challenge: hardware/software platform, telecommunications, and dealing with time differences. Dialectics as well as consensus around key questions emerged as discussion not only involved dealing with issues associated with the IOF conference but extensions to use in broader organizational contexts.

\subsection{Hardware/Software platform}

Platform challenges focused on how to resolve different hardware platforms of different conference sites, different software platforms, and different communication facilities in different countries. It was especially noted that the same interface across software platforms and network connections is needed so that as people move from work to home to traveling to conferences they can log in and see the same interface and metaphors they are used to getting work done. Of concern was providing a ubiquitous interface to a dynamic group memory i.e., all participants need to have equal access to all media. The World Wide Web in general and Netscape in particular provide only part of the answer. Standard software suites are important as is consistency of technical formats e.g., all slides via PowerPoint, etc. Of particular concern, however, was the ability to cover a wide range of technological capability. A basic dialectic and tension emerges as, on the one hand, we want universal capability but, on the other hand, we recognize that tolerance for a wide range of technological capability creates a multitude of integration problems.

There are two dimensions to this issue, simplicity versus complexity and standardization versus accommodation. Some participants favored going for simple solutions given limited economic conditions at most universities stating that simple but high quality solutions are best for now e.g., a high quality conference phone. Others noted, however, that it is in our best interest to explore the forefront of technology. Standardization tended to favor Microsoft Windows. As one participant noted, "I would suggest: Windows machine, some similar software interface for downloading/viewing files, searching, adding/responding to comments, accessing information about event times, on-line "socializing", and Internet access." Other participants lamented the rigidity this brings about and the desire for a mix of high tech, low tech, and no tech solutions e.g., coexistence between conventional and electronic technology for presentations. The following matrix summarizes participant discussion around this key question of simplicity versus complexity and standardization versus accommodation.

$$
\begin{aligned}
& \text { simple - standardization } \\
& \text { [boring] }
\end{aligned}
$$

simple - accommodation [workable - but limited]

$$
\begin{aligned}
& \text { complex standardization } \\
& \text { [interesting - Bill Gates smiles] }
\end{aligned}
$$

complex accommodation [chaos] 


\subsection{Telecommunications}

Multiple communications channels are required to not only support the more formal aspects of the IOF but also to support side conversations, spontaneous break-out sessions, etc. Unfortunately, the technology is not mature enough. As one participant noted, "the experiences trying to extend desktop videoconferencing systems to events distribution have been a complete failure when you transmit more that one face in $2 \mathrm{~B}+\mathrm{D}$." At this point, true multi-point video is but a dream. Bandwidth limitations and their impact on communication richness remain an important research issue. Much conversation revolved around the question "Why don't we just give up on video conferencing?"

- Some participants noted that there "doesn't seem to be too much progress in the last 10 years" and that "video conferencing has been given a long time to develop with little perceived progress." Others noted that "it seems like the payoffs are questionable and the costs are large. Can't the 'getting to know and trust you' phases be accomplished through computer conferencing? I hear all about these 'Internet Relationships'. With the low cost systems and even high-cost systems the distractions can be worse than if you had only audio. Further the Internet community is taking over the videoconferencing standardization issues anyway."

- However it was that noted that "actually there is great progress in the areas of video compression and the ability to send video over the Internet. Also new capabilities like holographic images are opening up new possibilities." Further, "Getting to know and trust people can be done via the Internet only to some extent. Computing conferencing is ONLY one of several layers need to actually accomplish work. Conferences are usually too unstructured and ad-hoc to build working teams that can accomplish real tasks and develop a sense of team identity." Video conferencing not only helps in the development of trust and assuring presence but is an effective way of communicating with people that you already know.

The session participants had a number of suggestions and conclusions to this key question of audio versus video versus data. Among them was the need to insure that video conferencing quality is adequate to the task. As one noted, "Proshare does not suffice for most needs." A suggestion to "create the listener category for people that prefer to 'attend' in a passive way" was responded to as follows: "This is a good idea. But rather than just one or several categories, there needs to be a continuum of control by the leader or leaders so that different options and privileges can be turned on and off for specific participants or subgroups of participants. Perhaps some of the features could be controlled by the participants themselves, at the discretion of the leader or the group as a whole through developing protocols that are appropriate to the nature of the task and the nature of the group."

\subsection{Dealing with time differences}

With a virtual conference, many time considerations become apparent e.g., "does the conference ever end and it can begin anytime, like now. "Participants need to apriori know the schedule of events so they can make plans to be involved in those activities that are motivating to them. There was a tension over how to schedule and degree of synchronous versus asynchronous interaction. One noted that "I find that with people in various time zones you do a lot of asynchronous work -catching up on what was put in by the group while you were sleeping." There was a suggestion that 
we "may want to try ignoring time differences and let some participate on an adhoc basis. This is what happens in the real world." Participants especially noted the need for integration of synchronous and asynchronous tools and that "we are using new technology to implement an old process. The old process was developed from different motivating factors and using different technology. Lets take off our blinders and think outside the box" to address this key question of the balance of synchronous and asynchronous interaction.

Group memory was an especially important item of discussion. In response to a comment that group memory must be kept current and well organized, a participant noted "Yes, this is key. One method to do this is to organize the information as it is created by using tools that allow one to build tasks, subtasks, and categories for different ideas as they are generated. The idea of using some search engine to sift through huge amounts of data only takes you so far. We need tools that let you organize and synthesize as you go through a process to accomplish an objective." Another noted that "memory contains structured and unstructured information such as documents and video and audio. We need tools that do that." In response to "there is a big power in defining categories and structure for memories," it was noted that "Correct. Somehow, the group needs to get some consensus on what is stored in the memory and how it is stored." It was suggested to use Lotus Notes as a good organizer and document database and InterNotes to insure ubiquitous access.

\section{CONCLUSION}

Participants in general concluded that we need good planning, testing, rethinking, testing, rethinking, and then, maybe, the implementation will work and provide high quality. As one noted, "Remember - the conference is the network or at least highly effected by the capability the network provides." The control of this very heterogeneous communication infrastructure makes it more difficult to run the conference. A participant noted that, in organizational contexts, "You need tools that allow you to pose ideas, argue about them, and determine the level of group consensus before the meeting. This allows actual meaningful work to be done during the meeting, rather than wasting lots of time level setting and getting issues on the table for the first time. This also allows people to think through issues thoroughly prior to a meeting and gather relevant information which they can bring to the meeting and share with others in real time."

Comments also suggested that more meaningful, long-term working relationships are created through long-term electronic communication and collaboration than through casual talk at a cocktail party. There was, however, a plea that "couldn't we have some kind of on-line, real-time(?) social times for people with similar research interests to allow them to get acquainted?" Concluding comments reiterated the importance of the need for redundancy and backup and disaster planning to accommodate reliability problems and that tech support during the conference at each site is critical. 